\title{
Contabilidad popular. Alternativa para resignificar las prácticas contables en las organizaciones sin ánimo de lucro. Estudio de caso
}

\author{
Popular accounting. Alternative to redefine accounting practices \\ in non-profit organizations. Case study
}

Recibido: 10-07-2020 • Aprobado: 24-02-2021 • Página inicial: 201- Página final: 222

Jennifer Andrea Vélez Bedoya*

Cristian Alonso Holguín Arenas**

\begin{abstract}
Resumen: el artículo presenta las características de la contabilidad popular como alternativa para reconocer y resignificar las prácticas contables de la Corporación Comité Universitario de Derechos Humanos Gustavo Marulanda de Medellín (CCUDDHHGM). Para su desarrollo, se reconoció el contexto en el cual surge la Corporación; adicionalmente, se realizó una caracterización de las prácticas y necesidades contables presentes en esta; y finalmente se estableció una aproximación conceptual a la contabilidad popular. Esta se fundamentó en la educación popular y sitúa a la contabilidad popular más allá del contexto de la economía solidaria. El enfoque investigativo es cualitativo y el método empleado fue el dialógico.
\end{abstract}

Palabras clave: contabilidad popular; educación popular; organizaciones de derechos humanos; prácticas contables.
Abstract: The aim of this article is to present the characteristics of Popular Accounting as an alternative to recognize and resignify the accounting practices of the Gustavo Marulanda University Human Rights Committee. For its development, it recognized the context in which the Corporation emerged. In addition, a characterization of the accounting practices and needs of the Corporation was carried out to finally make a conceptual approach to popular accounting. This is based on popular education and places popular accounting beyond the context of solidarity economy. The research approach is qualitative and the dialogical method was used.

Keywords: Popular accounting; popular education; human rights organizations;

\footnotetext{
* Contadora pública e integrante del Semillero de Investigación CONTAME del Tecnológico de Antioquia, Colombia jeanvlz2017@gmail.com ORCID: https://orcid.org/0000-0002-5235-8464

** Contador público e integrante del Semillero de Investigación CONTAME del Tecnológico de Antioquia, Colombia. alonsoholguinarenas1996@gmail.com ORCID: https://orcid.org/0000-0003-1882-8255
} 


\section{Contexto verez, s.y Holoum c.}

\section{Contabilidade popular. Alternativa para redefinir as práticas contábeis em organizações sem fins lucrativos. Estudo de caso}

Resumo: o artigo apresenta as características da contabilidade popular como alternativa para reconhecer e ressignificar as práticas contábeis da Corporación Comité Universitario de Derechos Humanos Gustavo Marulanda de Medellín (Corporação Comitê Universitário de Direitos Humanos Gustavo Marulanda de Medellín em português). Para o seu desenvolvimento, foi reconhecido o contexto em que a Corporação surge; adicionalmente, foi realizada uma caracterização das práticas e necessidades contábeis presentes nesta; e, finalmente, estabeleceu-se uma abordagem conceitual para a contabilidade popular. Baseia-se na educação popular e situa à contabilidade popular para além do contexto da economia solidária. A abordagem da pesquisa é qualitativa e o método utilizado foi dialógico.

Palavras-chave: contabilidade popular; educação popular; organizações de direitos humanos; práticas contábeis. 


\section{Introducción}

El presente artículo hace parte del proyecto de investigación Extensión solidaria. Prácticas Contables para las organizaciones desde una propuesta de contabilidad popular, el cual tiene como objetivo proponer la contabilidad popular como alternativa para resignificar las prácticas contables en las organizaciones de economía solidaria-alternativa de Colombia. El proyecto está adscrito a la línea de investigación en Contabilidad del Grupo de Investigación Observatorio Público, de la Facultad de Ciencias Administrativas y Económicas de la Institución Universitaria Tecnológico de Antioquia.

Uno de los fines de la academia es contribuir a la construcción de conocimiento a través de la praxis en contextos sociales diversos, y debido a la multiplicidad de formas organizativas en las que puede accionar el saber contable, ha surgido entre los integrantes del Semillero Contame ${ }^{1}$ el cuestionamiento sobre las dinámicas y ejercicios de la práctica contable en organizaciones de carácter no tradicional, las cuales desarrollan actividades económicas alternas en el contexto de la economía solidaria.

En este sentido, se comenzó a cuestionar desde este espacio académico e investigativo el desarrollo conceptual de una categoría en contabilidad con la cual ese otro tipo de organizaciones tuviera el reconocimiento de sus prácticas. Dicha categoría es la contabilidad popular, la cual surge a partir de la metodología de círculos solidarios, definidos como "[...] una estrategia que tienen diferentes nombres en otras partes del mundo, [pero] constituyen la materialización de las apuestas de economía solidaria" (Rodríguez et al., 2018, p. 412). Es decir, son estrategias económicas alternas para aquellas comunidades con condiciones materiales poco favorables y, de forma puntual, han sido desarrolladas con un componente social, político y cultural potente.

La aproximación conceptual a esta categoría ha sido trabajada con anterioridad por las docentes de la Institución Universitaria Tecnológico de Antioquia: Yuliana Gómez Zapata, María Alejandra Rodríguez Triana y Natalia Andrea Gallón, y de forma más reciente por los integrantes del semillero Contame de la misma institución, así como estudiantes de otras instituciones de educación superior que se han vinculado al proceso, entre las que se encuentran la Universidad de Antioquia y el Politécnico Colombiano Jaime Isaza Cadavid.

\footnotetext{
'Fundado en 2013, adscrito a la Facultad de Ciencias administrativas y económicas del Tecnológico de Antioquia y coordinado por la profesora Yuliana Gómez Zapata.
} 


\section{Contexto verez, s.y Holoum c.}

La puesta principal ha estado direccionada hacia el desarrollo teórico-conceptual de esta categoría contable emergente, a partir del trabajo experiencial desarrollado con diversas organizaciones, con las cuales se configuran un conjunto de prácticas sociales, organizacionales y contables que pueden ser potenciadas desde la orientación y posibilidades que les puede brindar esta categoría, en la cual el elemento pedagógico y político resulta ser esencial.

Por tal motivo, en el desarrollo de esta investigación, se cuestiona acerca de cuáles son las características que posee la contabilidad popular, con las cuales sería posible reconocer y, a su vez, resignificar las prácticas contables existentes en una organización dedicada a la promoción y defensa de los Derechos Humanos, como es el caso de la Corporación Comité Universitario de Derechos Humanos Gustavo Marulanda (CCUDDHHGM), la cual se presenta como el tipo de organización en donde puede circunscribirse el campo de acción de la contabilidad popular, ya que su carácter organizacional cuenta con un componente político de transformación social. Además, es en un campo que no ha sido abordado con anterioridad en el desarrollo de esta categoría contable.

En ese sentido, la multiplicidad de formas de organización constituye un foco importante de desarrollo en esta investigación, así como un reto para la contabilidad popular, puesto que, para cada una de estas organizaciones, es necesario el reconocimiento de sus particularidades, en torno a sus prácticas contables y la gestión organizacional. En el caso de la CCUDDHHGM no es la excepción, puesto que se encuentra categorizada en un tipo formal de organización y regida por determinada normatividad contable y tributaria. No obstante, dentro de sus dinámicas estos elementos no han sido priorizados, por lo cual resulta útil la aplicación de la contabilidad popular, dado que se puede potenciar sus prácticas como organización y desarrollar de forma más óptima su objeto social.

Por tal motivo, el objetivo de la presente investigación, es presentar las características de la categoría contabilidad popular como una posible alternativa para reconocer y resignificar las prácticas contables de la CCUDDHHGM. Por lo tanto, se reconoce, en primer lugar, el contexto en el cual surge la Corporación, así como su situación actual, para identificar los hechos que motivaron el desarrollo de su labor social y sus particularidades organizativas. En segundo lugar, se busca caracterizar las prácticas y necesidades contables presentes en la CCUDDHHGM, a partir de la interacción con los diferentes agentes que la integran. En tercer lugar, se pretende realizar una aproximación conceptual a la contabilidad popular partiendo de las necesidades y prácticas contables identificadas en la corporación, así como de la fundamentación teórica consultada. 
Lo anterior denota un interés por desarrollar una línea de investigación en contabilidad popular, la cual es potencial a la hora de encontrar escenarios diferentes en donde el saber y la praxis contable tengan la posibilidad de actuar y, a su vez, los procesos y experiencias desarrollados por este otro tipo de organizaciones nutran el desarrollo de construcción de conocimiento conjunto en el ámbito contable, administrativo y organizacional. Se aporta así no solo a la esfera académica, sino también la dinamización de los procesos en ese tipo organizaciones, que tienen un impacto social, económico político y cultural en los territorios en donde se desarrolla su objeto social.

La estructura del artículo comprende un primer apartado que revisa la literatura sobre contabilidad popular, la cual se constituye de los antecedentes de la investigación, seguido de la descripción de la metodología empleada y el desarrollo de los objetivos específicos, partiendo desde la descripción del contexto en el cual surge la corporación y su situación actual. En un segundo apartado se detalla las prácticas y necesidades contables detectadas a través del proceso de interacción con la corporación. El tercer formula la aproximación teórica conceptual de la categoría de contabilidad popular, partiendo inicialmente desde el contexto de la economía solidaria, en donde se reconoce a los círculos solidarios como la estrategia económica con la cual surge esta categoría; así mismo, la fundamentación teórica consultada y el proceso reflexivo llevado a cabo en el transcurso de la investigación.

\section{Antecedentes}

El primer acercamiento a la categoría de contabilidad popular se encuentra en las publicaciones de Rodríguez et al. (2016; 2018), quienes exponen algunos planteamientos sobre el papel de la contabilidad en propuestas de economía solidaria y alternativa, que se han desarrollado en organizaciones de carácter social y popular. Específicamente, emerge del trabajo realizado con formas organizativas que se viene gestando en el escenario local, como son los círculos solidarios y los circuitos de producción campesina y comercio justo. Para ello, se realiza una descripción del contexto en el cual surgen este tipo de propuestas, para, posteriormente, adentrarse al tema de la contabilidad popular y alternativa.

Se profundiza en las alternativas de círculos solidarios desarrollados en la Corporación para el Fomento de las Micro finanzas y el Microcrédito Fomentamos, de la ciudad de Medellín. Se observó cómo las personas pertenecientes aumentaron su empoderamiento frente a temas como la realización de las cuentas de sus negocios y el desarrollo de la capacidad de involucrarse con otras personas que comparten intereses y problemáticas comunes (Rodríguez et al., 2018). Es así como 


\section{Contexto veser, y.ytougu, c.}

se expone que, al interior de estas propuestas, se produce un tipo de información contable y financiera cuyas particularidades deben ser comprendidas desde otra óptica. Por tanto, la contabilidad cobra vital importancia en este proceso, debido a que no puede ser vista de manera lineal y estrecha, como se hace comúnmente en la corriente ortodoxa.

De manera más reciente, Gallón et al., (2020) realizan una caracterización de los problemas y necesidades contables de las organizaciones de economía solidaria de los ex combatientes de las FARC-EP para, posteriormente, realizar una aproximación teórica-conceptual de la contabilidad popular. De acuerdo con las autoras, la contabilidad popular es una

práctica pedagógico-política y social que busca reafirmar y resignificar los conocimientos y prácticas contables que las organizaciones desarrollan para responder a sus necesidades y generar las condiciones para la emergencia de otras prácticas contables situadas con las organizaciones de economía solidaria (Gallón et al., 2020, p. 38).

Este argumento se encuentra fundamentado en la educación popular como eje transversal de las relaciones entre la contabilidad con los procesos y fines organizativos, además de la relación de esta misma con la experiencia y conocimientos propios de los sujetos que componen las organizaciones.

Posteriormente, en el trabajo de Gallego-Ruiz (2019) se retoma ciertas concepciones iniciales de la aproximación categórica de la contabilidad popular, al indicarse, en primer lugar, el contexto organizacional en el cual se hace presente, haciendo referencia a las organizaciones de carácter no parametral, es decir, aquellas organizaciones cuyo objeto social no se suscribe a la lógica de la rentabilidad convencional, ya que la rentabilidad en este tipo de organizaciones se traduce además en posibilidad de continuidad y prevalencia de su gestión social en el tiempo.

En ese sentido, la autora hace referencia a las entidades sin ánimo de lucro, las organizaciones solidarias, las cooperativas, organizaciones de procesos sociales y culturales, entre otros. Adicional a esto, tiene en cuenta un aspecto clave y común en las organizaciones anteriormente mencionadas: la dificultad en la gestión de recursos que limita el desarrollo del objeto social. En este punto es donde surge la necesidad de dotar de un nuevo significado los procesos contables, de acuerdo con las condiciones que presentan las organizaciones (Gallego-Ruiz, 2019).

En síntesis, la categoría contabilidad popular es un concepto poco explorado en el ámbito investigativo, el cual emerge como una alternativa para resignificar las 
prácticas contables en organizaciones de distinta índole, no solo las propuestas de economía solidaria y alternativa, sino también en formas organizativas de carácter social, comunitario, de derechos humanos y demás, en las que el componente político se convierte en el eje central de su accionar.

\section{Metodología}

Para el desarrollo de la presente investigación se optó por el método dialógico, mediante el cual se posibilita la construcción de conocimientos a través de la resignificación de la práctica social en escenarios que distan de la neutralización y mercantilización de conocimientos estandarizados y válidos únicamente desde un ámbito académico. En ese sentido, el espacio propuesto se nutre de la diversidad y riqueza interpretativa de los hechos que transcurren desde la cotidianidad, es decir, un conocimiento que se construye entre dos sujetos con la mediación del mundo (Freire, 2005).

En investigaciones de carácter socioeducativo, psicosocial o sociocultural, caracterizados por la reflexividad, la dialogicidad y solidaridad, se recuperan y resignifican construcciones colectivas de conocimiento solidario que permiten reconocer y expresar la posibilidad de producir conocimientos y discursos sociales en condiciones experienciales diferentes (Ghiso, 2016).

El enfoque de la investigación es cualitativo, caracterizado por el rechazo a la pretensión racional de solo cuantificar la realidad humana, en cambio da importancia al contexto, a la función y al significado de los actos humanos, valora la realidad como es vivida y percibida, con las ideas, los sentimientos y las motivaciones de sus actores (Mesías, 2010, p. 1).

Ahora bien, para el desarrollo de los objetivos propuestos, se establecieron unas categorías de análisis, que obedecieron al elemento investigativo central de cada uno de ellos, siendo respectivamente: la Corporación Comité de Derechos Humanos Gustavo Marulanda de Medellín, como primera categoría; las prácticas y necesidades contables presentes en dicha corporación, la segunda categoría; como tercera categoría se estableció a la contabilidad popular, como el eje principal de desarrollo conceptual de la investigación.

Así mismo, se emplearon fuentes documentales que permitieron realizar una fundamentación y contextualización teórica preliminar. Y como fuentes orales se seleccionaron a expertos temáticos y prácticos: el historiador Alejandro Sierra Urrego, docente de la Institución Universitaria Tecnológica de Antioquia, el cual 
cuenta con una amplia experiencia en el ámbito de derechos humanos, siendo a su vez fundador de la CCUDDHHGM; y a Isaac Buitrago, abogado y miembro actual de la Corporación. En ese sentido, la selección de la unidad de análisis se realizó a criterio y a conveniencia.

Adicionalmente, se emplearon el rastreo bibliográfico, la conversación y el diagnóstico, como técnicas; a su vez, se utilizaron la matriz bibliográfica, el relato, la matriz de códigos y categorías y la matriz de situación contable, como instrumentos de recolección y generación de información.

\section{Tabla 1}

\section{Estrategia metodológica}

\begin{tabular}{|c|c|c|c|c|c|}
\hline Objetivo específico & $\begin{array}{l}\text { Categorías de } \\
\text { análisis }\end{array}$ & $\begin{array}{l}\text { Fuentes de } \\
\text { información }\end{array}$ & $\begin{array}{l}\text { Selección de } \\
\text { unidad de } \\
\text { análisis }\end{array}$ & $\begin{array}{l}\text { Marcos sociales } \\
\text { de interacción }\end{array}$ & $\begin{array}{l}\text { Instrumentos } \\
\text { de recolección } \\
\text { y generación de } \\
\text { información }\end{array}$ \\
\hline \multirow[b]{2}{*}{$\begin{array}{l}\text { Reconocer el contexto en } \\
\text { el cual surge la Corpora- } \\
\text { ción Comité Universitario } \\
\text { de Derechos Humanos } \\
\text { Gustavo Marulanda de } \\
\text { Medellín, así como su si- } \\
\text { tuación actual, para identi- } \\
\text { ficar, tanto los hechos que } \\
\text { motivaron el desarrollo de } \\
\text { su labor, como sus particu- } \\
\text { laridades organizativas. }\end{array}$} & \multirow[b]{2}{*}{$\begin{array}{c}\text { Corporación } \\
\text { Comité Univer- } \\
\text { sitario de Dere- } \\
\text { chos Humanos } \\
\text { Gustavo Maru- } \\
\text { landa. }\end{array}$} & Documentales. & A conveniencia. & $\begin{array}{c}\text { Rastreo } \\
\text { bibliográfico. }\end{array}$ & Matriz bibliográfica. \\
\hline & & $\begin{array}{l}\text { Expertos } \\
\text { prácticos y } \\
\text { temáticos: } \\
\text { fundadores } \\
\text { y miembros } \\
\text { actuales de la } \\
\text { corporación. }\end{array}$ & A criterio. & Conversación. & $\begin{array}{c}\text { Relato. } \\
\text { Matriz de códigos y } \\
\text { categorías. }\end{array}$ \\
\hline \multirow{2}{*}{$\begin{array}{l}\text { Caracterizar las prácticas } \\
\text { y necesidades contables } \\
\text { presentes en la Corpora- } \\
\text { ción Comité Universitario } \\
\text { de Derechos Humanos } \\
\text { Gustavo Marulanda de } \\
\text { Medellín, a partir de la in- } \\
\text { teracción con los diferen- } \\
\text { tes agentes que integran la } \\
\text { organización. }\end{array}$} & \multirow[b]{2}{*}{$\begin{array}{l}\text { Prácticas y } \\
\text { necesidades } \\
\text { contables. }\end{array}$} & \multirow[b]{2}{*}{$\begin{array}{l}\text { Fundadores } \\
\text { y miembros } \\
\text { actuales de la } \\
\text { Corporación. }\end{array}$} & \multirow[b]{2}{*}{$\begin{array}{l}\text { A conveniencia. } \\
\text { Conversación. }\end{array}$} & Diagnóstico. & $\begin{array}{l}\text { Matriz de situación } \\
\text { contable. }\end{array}$ \\
\hline & & & & Relato. & \\
\hline \multirow{2}{*}{$\begin{array}{l}\text { Realizar una aproximación } \\
\text { conceptual a la contabili- } \\
\text { dad popular partiendo de } \\
\text { las necesidades y prácticas } \\
\text { contables identificadas en } \\
\text { la corporación, así como } \\
\text { de la fundamentación teó- } \\
\text { rica consultada. }\end{array}$} & \multirow[b]{2}{*}{$\begin{array}{c}\text { Contabilidad } \\
\text { popular. }\end{array}$} & $\begin{array}{l}\text { Miembros } \\
\text { actuales de la } \\
\text { Corporación. }\end{array}$ & A conveniencia. & Conversación. & Relato. \\
\hline & & Documental. & A conveniencia. & $\begin{array}{c}\text { Rastreo } \\
\text { bibliográfico. }\end{array}$ & Matriz bibliográfica. \\
\hline
\end{tabular}

Elaboración propia (2020). 


\section{Contexto del surgimiento de la Corporación Comité Universitario de Derechos Humanos Gustavo Marulanda}

El contexto en el que surge la CCUDDHHGM está enmarcado en el proceso de negociaciones de Tratado de Libre Comercio (TLC) entre Estados unidos y Colombia: unos meses después del anuncio oficial, por parte de Estados Unidos, de la intención de iniciar las negociaciones de un TLC con Colombia, Ecuador y Perú, se inició la serie de rondas de negociación que tendrían fecha entre el 18 de mayo de 2004 y el 22 de julio de 2005 (Ministerio de Comercio, industria y Turismo, s. f.).

Ante esta situación, el 10 de febrero de 2005, se dio una jornada nacional de protesta en contra de las mencionadas negociaciones que se enmarcan en un ciclo de manifestaciones tanto en rechazo al TLC como al referendo reeleccionista de Álvaro Uribe, las cuales se venían desarrollando desde 2003 (Archila, 2012). En Medellín, la protesta tuvo lugar en la Universidad de Antioquia.

Al respecto, Alejandro Sierra (Comunicación personal, 3 de marzo de 2020) indicó que en dicha universidad se organizó una jornada de protesta muy fuerte. No obstante, las cosas no salieron como se pensaron, debido a que se presentó una explosión entre los estudiantes que protestaban. En medio de esto, por lo menos 17 estudiantes resultaron heridos (Caracol Radio, 2005).

Posterior a ese suceso, se abrió una investigación en la Universidad de Antioquia, a cargo de la fiscal 51 Fabiola Mejía Muñetón, lo que originó que el 4 de mayo de ese año, se diera una captura masiva de 14 estudiantes (El Tiempo, 2005), entre los que se encontraban, no solo participantes de las protestas, sino también líderes estudiantiles.

En medio de toda esta situación, surgió un comité de solidaridad con la intención de ayudar a las personas que se encontraban detenidas y reunir así recursos para los 14 detenidos, en especial para cuatro de ellos que habían resultado heridos en medio de la protesta y aún tenían quemaduras en su piel; por lo tanto, requerían operaciones para hacer injertos de piel y cuidados paliativos (Sierra, comunicación personal, 03 de marzo de 2020).

Una vez creado dicho comité, este fue impulsado por dos estudiantes, los cuales presionaron a la universidad para integrar a un representante; a su vez, algunos profesores de derecho, por iniciativa propia, decidieron vincularse al proceso. Con el propósito de garantizar un mínimo de cien mil pesos semanales a cada uno de los estudiantes detenidos, la primera tarea que se propuso el Comité fue realizar 


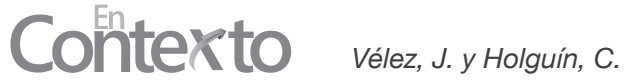

la recolección de dinero, recurriendo a la ayuda de sindicatos, profesores y demás personas que manifestaron su apoyo.

Sin embargo, debido a diversas situaciones, la constitución legal de su actividad se convirtió en una obligación. En primer lugar, porque la fiscal Fabiola Mejía Muñetón adelantó investigaciones en contra de los miembros del comité "porque no comprendía qué era eso de la solidaridad y consideraba que, si había personas recogiendo dinero para llevarlos a ellos, es porque era sus cómplices" (Sierra, comunicación personal, 03 de marzo de 2020).

En segundo lugar, porque al momento de solicitar apoyo de la Universidad de Antioquia, la oficina jurídica argumentó que, debido a la regulación existente, no podía brindarle ese tipo de apoyo a personas que se decían ser parte de un comité. Por esta razón, decidieron estudiar cuál figura jurídica era más conveniente, entre una fundación, una corporación o una asociación.

Por otro lado, crear una corporación traía ciertos beneficios, entre ellos, abrir una cuenta de ahorros de la corporación para recibir allí los dineros de solidaridad, que se destinaba no solo "al apoyo económico de los estudiantes detenidos, sino también al acompañamiento a marchas, pequeñas pautas y diciéndole a la gente cuáles son los límites de la fuerza pública" (Sierra, comunicación personal, 03 de marzo de 2020).

Fue así como surgió la necesidad de crear una organización de derechos humanos, bajo la figura de Corporación, a la cual se optó por darle un nombre reivindicativo, de un estudiante que había sido asesinado en su momento seis años atrás: Gustavo Marulanda García, asesinado a las afueras de la Universidad de Antioquia.

Actualmente la Corporación se encuentra en espera de definir su personería jurídica, debido a situaciones que se describirán más adelante. Razón por la cual sus actividades como organización se encuentran paralizadas.

\section{Prácticas y necesidades contables}

En el presente apartado se presenta una descripción de las prácticas y necesidades contables, que se detectaron en los ejercicios de interacción con la corporación. Lo anterior se logró mediante una conversación con uno de sus actuales integrantes, en la que se abordaron temas como la situación actual de la CCUDDHHGM en el ámbito administrativo y contable, en donde se aplicaron además dos matrices construidas con el fin de identificar dichas prácticas y necesidades: la primera 
permitió establecer un panorama del manejo de recursos, así como el de sus deudas, ingresos y gastos. Por su parte, con la segunda preguntó por el estado de la corporación en términos de requisitos legales y contables, el avance de esos trámites y algunas observaciones.

Este ejercicio de interacción con la corporación permitió observar además ciertas prácticas que son necesarias destacar: las reuniones mensuales, los informes que se presentan en dichas reuniones, la gestión de la información de la corporación, la toma de decisiones y la preocupación por estar al día en los asuntos en materia legal.

En cuanto a uno de los canales de comunicación de la corporación: las reuniones mensuales constituyen un medio importante para discutir diversos asuntos; uno de los temas de la reunión es informe del valor de los gastos necesarios para llevar a cabo alguna actividad que se haya decidido apoyar. Lo anterior muestra cierto interés por evidenciar cada transacción de manera transparente y la comunicación en la organización.

Adicionalmente, los informes que se hacen a los demás agentes de la Corporación están marcados por la confianza. Esto constituye un punto de partida interesante para analizar la forma en que la contabilidad actúa puesto que, aunque no hay un informe escrito, detallado y con todos los requisitos legales, existe una conciencia sobre el manejo del dinero y a quiénes puede ser útil la información.

Otra práctica presente en la corporación está relacionada con el manejo de la información que esta posee, puesto que todo documento o elemento que pueda ser de gran importancia para esta, se encuentra recopilada en un almacenamiento en la nube, constituyendo así un sistema de información que obedece a sus necesidades y que está al alcance de todos los que la integran.

En la Corporación, así como en los movimientos sociales en general, la toma de decisiones se caracteriza por la horizontalidad en la deliberación y decisión; de esta manera, figuras de jerarquía como el presidente de la junta directiva se utiliza más para espacios de interlocución con la institucionalidad. Sin embargo, al interior de la organización, cada uno sin distinción tiene el derecho a recibir informes de cada una de las actividades y a participar en igualdad de condiciones en los debates internos (Buitrago, Comunicación personal, 20 de mayo de 2020). Por último, cabe resaltar que los trámites ante cámara de comercio y otros de carácter legal, se mantienen al día y con todos los requisitos expresados en la ley. 


\section{Contexto veser, y.ytougu, c.}

En términos generales, las principales necesidades de la corporación van encaminadas hacia tres direcciones, relacionadas con lo tributario, administrativo y contable. En primer lugar, los asuntos tributarios hacen referencias a Registro Único Tributario (RUT), Régimen Tributario Especial y declaraciones tributarias. El incumplimiento de estas obligaciones podría incurrir en sanciones que, teniendo en cuenta la realidad económica de la organización, sería un egreso que la corporación no estaría preparada para asumir.

En términos administrativos se plantean proyectos relacionados con la sostenibilidad financiera que les permita, entre otros, contratar mínimo un funcionario que esté pendiente de las fechas, vencimientos, obligaciones y demás asuntos de índole administrativo de la corporación. Para garantizar la sostenibilidad, tienen como proyecto buscar tres líneas de financiación: recursos de cooperación internacional, tener la capacidad para contratar con el Estado "con criterios éticos y políticos relacionados con nuestro quehacer como defensores y defensoras de derechos humanos" (Buitrago, Comunicación personal, 20 de mayo de 2020) y consolidar un grupo de estudio que adelanta un proyecto que busca analizar las resoluciones de inclusión al registro único de víctimas, para presentarlo a una de las convocatorias BUPPE $^{2}$ de la Universidad de Antioquia.

Desde el momento de su surgimiento, la corporación ha pasado por varias transformaciones, se pretende que sea una organización de carácter nacional; sin embargo, y pese a las buenas intenciones, todavía carece de la posibilidad de generar la suficiente cohesión por diversos factores, ya sea que los territorios son muy disímiles o porque la corporación no produce recursos suficientes para pagar funcionarios o funcionarias; por tal motivo, se decidió que cada departamento trabaje bajo sus propias dinámicas. Por tanto, el autoconocimiento e identificación de los limitantes organizacionales son fundamentales para trazar los diferentes caminos, así como analizar diversas opciones que permitan a la corporación gestionar de forma más adecuada sus recursos humanos y económicos.

En la organización, las necesidades contables van encaminadas principalmente hacia el cumplimiento de exigencias de los entes de fiscalización y control, específicamente, los aspectos técnicos normativos que se deben tener en cuenta al momento elaborar un reporte financiero, tributario o de gestión. Así como el componente contable que dé cuenta de las actividades de la organización, reflejadas en transacciones de índole financiero. Sin embargo, en formas organizativas como esta no se encuentra un acompañamiento constante por parte de las entidades reguladoras que emiten tal normatividad, convirtiéndose en un obstáculo latente para la supervivencia de este tipo de organizaciones.

\footnotetext{
${ }^{2}$ Banco Universitario de Programas y Proyectos de Extensión: “[...] es el instrumento empleado por la Vicerrectoría de Extensión para canalizar y fortalecer las iniciativas de los diferentes actores universitarios" (Universidad de Antioquia, 2020).
} 
Por tal motivo, se requiere de la orientación de las prácticas existentes en la organización que les permita fortalecerse y, en un futuro, poder lograr todas las metas y sueños planteados. En este grupo de necesidades, se incluye el manejo de los gastos e ingresos, la realización de documentos soporte para que cada agente se entere del valor de los mismos (mes a mes). Adicionalmente, se requiere de un sistema o metodología para el registro y control, tanto de los activos fijos que posee la organización, como de los recursos y erogaciones que tiene el transcurso del desarrollo de sus actividades.

En Colombia, además del complicado contexto en que las organizaciones de Derechos Humanos se desenvuelven, también deben responder con una gran cantidad de retos, obligaciones y otras adversidades que pueden convertirse en serios inconvenientes. Por esto, el segundo grupo de necesidades está compuesto por la obligatoriedad de la presentación de Estados Financieros: informes a entes de control, actualización trámites de cámara de comercio, estar al día con la administración tributaria, buscar fuentes de financiación, mantenerse al día con seguridad social y parafiscalidad, entre muchos otros. Estas necesidades convierten en un verdadero reto de supervivencia a estas organizaciones.

Específicamente, la búsqueda de sostenibilidad financiera de las Organizaciones de Derechos Humanos es algo preocupante toda vez que, al tener que vivir de la autofinanciación, la competencia por los proyectos de cooperación internacional se convierte en una verdadera lucha darwiniana (Breton, 2001, como se citó en Gómez-Quintero, 2014). No obstante, las agencias de Cooperación Internacional establecen determinados requisitos para poder participar en sus convocatorias, relacionados con el manejo y reporte de la información financiera, entre otros. Como respuesta, algunas organizaciones desarrollan un departamento de contabilidad, otras con menos capacidad contratan servicios temporales y otras simplemente no encuentran solución. Por tal motivo, “[...] las grandes convocatorias se las llevan las grandes corporaciones de derechos humanos, pero las pequeñas organizaciones, las que trabajan con la base social, las que no tienen ese conocimiento [en términos administrativos y contables], no logran ese alcance" (Sierra, comunicación personal, 03 de marzo de 2020).

\section{Aproximación teórico-conceptual a la contabilidad popular}

La categoría contabilidad popular es una línea de investigación que está en construcción. Esta denomina un conjunto de prácticas contables que se articulan a una realidad concreta desde las múltiples expresiones socioeconómicas como la pobreza, la marginalidad y la economía de supervivencia (Rodríguez et al., 2018). 


\section{Contexto veser, y.ytougu, c.}

En ese sentido, esta categoría conceptual en contabilidad concibe como elemento esencial lo popular, lo cual es comprendido a su vez desde la educación popular y su relación con lo comunitario, lo barrial y con sectores desfavorecidos o excluidos. Se encuentra vinculada además con procesos de carácter político y emancipatorio, en los que la educación popular se constituye como un intento de desarrollar acciones intencionales orientadas a ampliar las formas de comprender y actuar de los sectores populares, es decir, hacia la generación de una subjetividad apropiada a la construcción de un proyecto político liberador (Torres, 1993, p. 15).

Es así como se puede connotar además lo "popular", en los dos sentidos que propone el sociólogo Helio Gallardo (2006). Por un lado, se basa en la noción de "pueblo social" (sectores sociales que sufren asimetrías de cualquier tipo: opresión, discriminación, exclusión, explotación, etc.); y por otro, en la noción de "pueblo político" (cualquier sector que lucha por eliminar dichas asimetrías).

Ahora bien, la contabilidad popular emerge en el contexto de la economía solidaria; y este contexto económico en términos de Restrepo et al. (2015) constituye un mecanismo alternativo de desarrollo, consistente en prácticas de autogestión y asociación comunitaria que permiten fomentar el progreso de sus participantes, basados en la participación autónoma del individuo en la construcción de su bienestar, el de su familia y la comunidad.

A su vez, y de forma contextuada, emerge en la metodología de los círculos solidarios, los cuales se presentan como una estrategia económica para aquellas comunidades carentes de recursos y capacidad de inversión suficiente, razón por la cual, dentro del sistema económico tradicional, se ha configurado un ambiente de exclusión, en torno a las necesidades y dinámicas sociales en las que están inmersas dichas comunidades que apuestan por el desarrollo de actividades económicas alternativas, apoyadas en un conjunto de valores: como lo son la solidaridad y la confianza.

Esta propuesta de economía solidaria ha sido desarrollada en la ciudad de Medellín por la Corporación Fomentamos, en la que los círculos solidarios se han constituido en un programa con una innovadora metodología de crédito, que tiene como objeto crear una modalidad interna, no solo de crédito, sino también de ahorro para las comunidades de escasos recursos, especialmente para las mujeres cabeza de familia. Estos buscan generar además mejores condiciones de vida en las personas que participan del programa a través del fortalecimiento de su negocio. Por lo cual ofrecen además servicios de capacitación, asesoría y acompañamiento para formación personal (Fomentamos, 2016).

Es importante señalar que, si bien este tipo de estrategias conviven con el actual modo de producción dominante -capitalista-, no corresponden completamente a su 
lógica. No son generadas por el Estado ni por el sector privado (formal); sin embargo, mantienen cierto tipo de relaciones con ellos, intentan elaborar alternativas de desarrollo (comunitario, local, regional) dentro de un contexto adverso y ambiguo: el de la globalización/exclusión (Diez, 2002, p. 22).

En ese punto, se considera importante destacar lo siguiente: la contabilidad como saber interdisciplinar tiene la posibilidad de aportar herramientas que, si bien en un primer momento pueden ser catalogadas con un índole técnico e instrumental, en el contexto comunitario son empleadas posiblemente sin percatarse de ello, bajo unas prácticas que se configuran desde lo cotidiano y experiencial. Por tal motivo, lo social y lo que es catalogado como financiero no parece estar contrapuesto. Por el contrario, este saber puede potenciar las comunidades bajo unas prácticas situadas en una realidad concreta, para que desarrollen de forma más óptima aquellas actividades económicas alternas, posibilitando además de la obtención un sustento, y la resignificación de sus prácticas comunitarias.

Una perspectiva distinta presenta Gallón et al. (2020) al señalar que el enfoque legal y financiero de la contabilidad, que prevalece en las organizaciones de economía solidaria, no permite lenguajes y representaciones de relaciones económicas colectivas y solidarias, en cambio sí medir e informar sobre la eficiencia y sostenibilidad económica como vectores, a su vez, de la eficacia administrativa y de la legitimidad del sector en la economía.

Dicha perspectiva difiere un poco de la aproximación categórica que está siendo construida en torno a la contabilidad popular, en tanto no se reconoce al elemento financiero y sus características inherentes de la contabilidad como disciplina económica y social, ya que se considera que las herramientas que ofrece lo financiero son necesarias e independiente del tipo de organización y contexto de desarrollo organizacional y que, sea bajo la óptica de economía tradicional o en el contexto de economía solidaria, esta puede constituirse en un medio para lograr los fines propuestos en las organizaciones.

De esa manera, se puede señalar que la contabilidad popular se circunscribe en un ámbito de organización no tradicional, o en términos de Gallego-Ruiz (2019) en organizaciones no parametrales, en tanto su campo de acción se encuentra en aquellas formas organizacionales que apuestan por el desarrollo de actividades económicas y sociales alternas, las cuales permiten generar dinámicas de resistencia, en torno a situaciones desfavorables concretas. Cuentan además con un componente de solidaridad alto, y en donde valores, como la confianza, incentivan los procesos de desarrollo comunitario, potenciando las habilidades organizativas de índole popular que apuntan a la construcción de una identidad colectiva. 


\section{Contexto verez, s.y Holoum c.}

Por tal motivo, se debe tener en cuenta que las dinámicas que se presentan en este tipo de organizaciones, sus prácticas y su concepción de la realidad, son aún más complejas puesto que en ellas se desarrolla un fuerte componente político de transformación social. En ese sentido, se puede apreciar que la contabilidad popular encuentra su fundamentación en la educación popular, haciendo referencia a "[...] aquellos procesos político-pedagógicos que buscan superar las relaciones de dominación, opresión, discriminación, explotación, inequidad y exclusión" (Jara-Holliday, 2010, p. 3).

Igualmente, lo manifiestan Gallón et al. (2020): [...] es a partir de la formación de los sujetos, la praxis, la concienciación y la reflexión crítica de la práctica, que se crean las posibilidades de comprensión y resignificación de las prácticas y el sentido contable en las organizaciones (p. 75).

Lo cual implica que el saber contable posibilita la configuración de conocimiento construido en conjunto con la comunidad, en los procesos llevados a cabo en torno a aquellas actividades económicas.

Por consiguiente, un elemento fundamental en la metodología de la educación popular, según lo expresa Moro (2004), es el papel que juega la teoría dialéctica del conocimiento, en donde a partir de la práctica, es decir, del saber popular de los actores sociales, de sus vivencias, de sus sentires, es posible el desarrollo de un proceso de teorización sobre las prácticas. Dicho proceso permite ubicar lo cotidiano e inmediato dentro de lo social y colectivo, por lo cual se considera que la construcción de conocimiento no puede desligarse de la práctica contextuada.

Tal situación, se presenta como una de las falencias o aspectos más cuestionados en el ámbito de la investigación, puesto que la construcción de conocimiento no debería presentarse como un proceso verticalizado entre el investigador y el sujeto o contexto de estudio. En ese sentido, en la educación popular, la contabilidad popular encuentra otro fundamento importante: busca establecerse relaciones de índole horizontal, democrática y de respeto mutuo entre los y las participantes. En ese caso, entre el profesional contable y la organización o comunidad, en busca de apoyo en la potencialización de sus prácticas cotidianas.

El conocimiento se genera en un proceso de interacción donde la experiencia y la subjetividad se construyen en el marco de una variedad de voces, condiciones y narrativas que sugieren el acto educativo como algo más que rechazos o acatamientos (Ghiso, 2016, p. 42). Allí mismo, el diálogo es el encuentro de los hombres para la tarea común de saber y actuar; es la fuente de poder desde su carga de criticidad y realidad contenidas en el lenguaje, las palabras y las interacciones. 
El diálogo es capacidad de reinvención, de conocimiento y reconocimiento (Ghiso, 2016, p. 43).

Otro elemento importante que nutre a la contabilidad popular, desde la fundamentación de la educación popular, es que busca promover el desarrollo integral de los involucrados en el proceso de construcción de su conocimiento, sus capacidades, sus habilidades, sus sueños; en definitiva, el afianzamiento del camino de búsqueda de identidad (Castillo et al., 1996). Es así como la identidad es un valor que buscan particularmente los sectores sociales desintegrados o excluidos por el sistema y que están ligados con los procesos de autoconciencia, con énfasis sobre la cultura y la formación de la comunidad (Moro, 2004).

Ahora bien, en el contexto organizacional en el que se desarrolló el presente trabajo de investigación, en torno a una organización con un compromiso político, como es la defensa de los derechos humanos, se abre camino hacia otra aproximación categórica de la contabilidad popular: si bien emerge del trabajo con economías de subsistencia, solidarias y alternativas, esta permite ampliar su espectro hacia otras economías que poseen, además de unas dinámicas sociales significativas, unas prácticas contables y organizacionales que deben valorarse y resignificarse.

En este sentido, se puede apreciar que en este tipo de organizaciones se encuentran dinámicas y prácticas que si bien no cuentan con una estructura formal o no están ceñidas completamente a legislación que las regula, el potencial con el que cuentan es inmenso y contribuye además a la transformación social de las comunidades en diferentes contextos y territorios, en los cuales la construcción de una identidad propia es supremamente vital, lo que constituye una de las principales apuestas del componente pedagógico, político y social de la contabilidad popular como alternativa para la resignificación de las prácticas existentes en las organizaciones.

En cuanto a las características de la contabilidad popular que fueron posibles identificar a través de la revisión de la fundamentación teórica y del proceso reflexivo de investigación, se encontró que estas se basan en la concepción de la contabilidad comprendida como una estructura estructurante, pues la forma específica como se re(presente) y proyecte la realidad organizacional, se deriva de unos intereses y necesidades que hacen que se configure, según los cambios de las necesidades de la información y los contextos sociales, culturales y políticos (Cardona-Hernández, 2013).

En este sentido, la adjetivación de popular connota una serie de elementos que tienen como eje transversal al elemento pedagógico y político con miras de emancipación y transformación social de los sujetos, puesto que la categoría de contabilidad popular encuentra su fundamentación principal en la educación popular. 


\section{Contexto verez, s.y Holoum c.}

Así mismo, en esta categoría se encuentra presente una intencionalidad vinculante entre el habitus, definiendo en términos de Bourdieu (2000) como "el sistema de disposiciones que es a su vez producto de la práctica y principio, esquema o matriz generadora de prácticas, de las percepciones, apreciaciones y acciones de los agentes" (p. 25), donde se configuran las prácticas de los actores sociales de las organizaciones haciendo diferente, por tanto, el contexto en que surgen sus relaciones.

Así como el proceso experiencial que ha forjado su capital social el "[...] cual está constituido por la totalidad de los recursos potenciales o actuales asociados a la posesión de una red duradera de relaciones de conocimiento y reconocimiento mutuo" (Bourdieu, 2000, p. 148). Y el habitus, en donde se genera las dinámicas del ejercicio del profesional contable y su capital cultural, en este caso institucionalizado, puesto que Bourdieu plantea que a través del título académico se confiere reconocimiento institucional al capital cultural poseído por una persona determinada.

$\mathrm{Su}$ apuesta, por tanto, es llevar a la praxis un conjunto de metodologías que se edifiquen en el reconocimiento del otro como parte del proceso de construcción de conocimiento, en donde el diálogo aparece como la forma de superar los fundamentalismos, de posibilitar el encuentro entre semejantes y diferentes (Freire, 1992, como se citó en Ghiso, 2013), puesto que en la academia y en el ejercicio profesional existen prejuicios y egos que posicionan el saber "formal" con superioridad sobre conocimientos que se construyen desde el empirismo y la práctica. Es así como la contabilidad popular puede caracterizarse por ser una categoría contable integradora, donde converge el conocimiento formal y el pragmático, reconociéndolos como iguales en términos de su valor e importancia, pues posibilita tener una concepción más amplia de la realidad organizacional.

Otro aspecto relevante de la contabilidad popular es su carácter orientador, donde su intencionalidad parte de brindar un acompañamiento a las organizaciones bajo una óptica de horizontalidad, y uno de sus retos más importantes constituye el comprender las dinámicas, los tiempos y las lógicas de las diversas formas asociativas, no solo en el contexto de la economía solidaria, sino en el entorno en donde se desarrollan las prácticas y actividades sociales, políticas y económicas de otro tipo de organizaciones en contextos diferentes. Configurando al igual un mundo aún más complejo que el de las organizaciones tradicionales.

La contabilidad popular hasta ahora no se encuentra dentro de las categorías contables existentes, como es el caso de la contabilidad social y de gestión, aunque claramente se nutre de elementos de estas, además no puede ser confundida con una asesoría organizacional convencional, puesto que su objetivo es contribuir a que las organizaciones identifiquen sus propios limitantes y elementos que 
obstaculicen sus dinámicas como organización. Así como posibilitar procesos de empoderamiento de las comunidades en torno a sus experiencias y saberes; y lo más significativo: lograr resignificar prácticas que son únicas e inherentes a su identidad como organización.

La contabilidad popular se encuentra vinculada fuertemente a una dimensión ética en la que los valores como la solidaridad y confianza direccionan las acciones de las organizaciones, generando el impacto y transformación social esperado por las comunidades y en donde la humildad, tolerancia y respeto son esenciales en la interacción con la organización orientada por el profesional contable o en proceso de formación.

Tales apreciaciones permiten vislumbrar que es necesario continuar con el proceso de aproximación categórica, porque la que logró construirse aún es incipiente y presenta además limitaciones, ya que uno de los obstáculos para el desarrollo del trabajo estuvo relacionado con el acercamiento e interacción con la corporación, por lo cual se recurrió a medios virtuales para lograr un acercamiento inicial, en el cual se pudo identificar ciertos aspectos de índole administrativo y contable que sirvieron para desarrollar una fase primaria con la corporación, que la fase de acompañamiento y orientación desde el ejercicio pedagógico contable, en el que se pudieran implementar un tipo de instrumentos y herramientas más interactivas, no pudo ser contemplado en el alcance del trabajo.

\section{Conclusiones}

La contabilidad popular se constituye como una categoría en construcción, la cual encuentra su fundamentación en la educación popular y emerge en un primer momento en el contexto de la economía solidaria con la metodología de círculos solidarios, que se presenta como una estrategia socioeconómica dirigida a comunidades vulnerables. No obstante, se pudo apreciar que su campo de acción puede abarcar otras formas organizativas, como es el caso de la Corporación Comité Universitario de Derechos Humanos Gustavo Marulanda, lo cual amplía su alcance y caracterización como una propuesta de resignificación de aquellas prácticas de índole contable que se encuentran inmersas en la dinámica de organizaciones consideradas como no tradicionales.

La Corporación presenta unas necesidades de índole contable y legal que limitan su operación y el desarrollo de su objeto social. Además, las prácticas contables propias, una vez reconocidas, son un punto de partida para la organización en su proceso de resignificación que incluye la emergencia de otras prácticas que obedecen a su devenir histórico, intereses, necesidades y, sobre todo, su pensamiento político. 


\section{Contexto veser, y.ytougu, c.}

Las características de la contabilidad popular que lograron identificarse, ubican a esta categoría contable con un potencial integrador y orientador, en donde el conocimiento considerado formal en términos de formación institucionalizada y el conocimiento que surge del proceso experiencial de las diversas maneras organizativas convergen con la intención de construir la posibilidad de resignificar aquellas prácticas que hace única su identidad.

Estas aproximaciones teórico-conceptuales iniciales aún son incipientes y parten de una intencionalidad investigativa que pretende continuar en proceso y que hace posible considerar a la emergente contabilidad popular como una categoría potencial en la visión y corriente heterodoxa de la investigación, que le permitiría al quehacer contable repensar su papel no solo en el ámbito académico, sino también en el campo de acción organizacional.

\section{Referencias}

Archila, M. (2012). El movimiento estudiantil en Colombia: una mirada histórica. Revista del Observatorio Social de América Latina, 13(31), 71-105. http:// biblioteca.clacso.edu.ar/clacso/osal/20120417105250/OSAL31.pdf

Bourdieu, P. (2000). Poder, Derecho y clases sociales. Desclée de Brouwer.

Caracol Radio. (11 de febrero de 2005). 17 heridos por explosión en la Universidad de Antioquia. Caracol. https://caracol.com.co/radio/2005/02/11/ nacional/1108107600_143874.html

Cardona-Hernández, J. (2013). Consideraciones de la contabilidad desde la teoría social de Pierre Bourdieu. Trazos para repensar la contabilidad desde la heterodoxia. Revista En-Contexto, 1(1), 193-213. https://ojs.tdea.edu.co/ index.php/encontexto/article/view/54

Castillo, L., Contreras, R., Duarte, C., y Valenzuela, G. (1996). Educación popular juvenil. Reflexiones desde la experiencia del colectivo de educación popular juvenil Newence. http:/www.cidpa.cl/wp-content/uploads/2013/05/4.8Valenzuela.pdf

Diez, A. (2002) ¿Qué son las llamadas “Economías Alternativas”? Millcayac, Anuario de Ciencias Politicas y Sociales, 1(1). https://bdigital.uncu.edu.ar/ objetos_digitales/125/DiezMillacayac.pdf

El Tiempo. (7 de mayo de 2005). 14 universitarios, detenidos por atentado. El Tiempo. https://www.eltiempo.com/archivo/documento/MAM-1672944

Fomentamos. (2016). Círculos Solidarios. http://www.fomentamos.com.co/ 
Freire, P. (2005). Pedagogía del Oprimido. Siglo Veintiuno Editores.

Gallardo, H. (2006). Revolución y cultura política popular en América Latina. Pasos, 125, 17-23. http://heliogallardo-americalatina.info

Gallego-Ruiz, M. (2019). IAP, contabilidad popular, sistematización de experiencias, caracterización de organizaciones no parametrales, prácticas contables y necesidades de la contabilidad popular [Tesis de pregrado, no publicada]. Tecnológico de Antioquia Institución Universitaria.

Gallón, N., Gómez Zapata Y., y Rodríguez, M. (2020). Contabilidad popular. Una alternativa socio-práxica para la resignificación de las prácticas contables en las organizaciones de economía solidaria de Colombia. Contaduría Universidad de Antioquia, 77, 37-79.

Ghiso, A. (2013). Sostener una mano o encadenar un alma: Legados de Paulo Freire para la Conceptualización de la Pedagogía Social en América Latina. Revista Contexto \& Educação, 15(59), 31-53. https://www.revistas.unijui. edu.br/index.php/contextoeducacao/article/view/1231

Ghiso, A. (2016). Reflexividad dialógica, como experiencia de epistemes sentipensantes y solidarias. El Ágora USB, 17(1), 255-264. https://doi. org/10.21500/16578031.2823

Gómez-Quintero, J. (2014). Organizaciones no gubernamentales y entidades sin ánimo de lucro en Colombia. Despolitización de la sociedad civil y tercerización del Estado. Gestión y Política Pública, 23(2), 359-384.

Jara-Holliday, O. (2010). Educación popular y cambio social en américa latina. http://www.iepala.es/IMG/pdf/CEAAL_Educacion_Popular_y_Cambio_ Social_en_AL-Oscar_Jara.pdf

Mesías, O. (2010). La Investigación Cualitativa. https://www.academia. edu/22351468/LA_INVESTIGACION_CUALITATIVA?auto=download

Ministerio de Comercio, industria y Turismo. (s. f.). La negociación del TLC de Colombia con los Estados Unidos. http:/www.tlc.gov.co/ getattachment/acuerdos/vigente/acuerdo-de-promocion-comercial-entrela-republ-1/contenido/documentos-y-enlaces-de-interes/documentos-dela-negociacion/la-negociacion-del-tlc-de-colombia-con-los-estados/lanegociacion-del-tlc-de-colombia-con-los-estados-unidos.pdf.aspx

Moro, W. (2004). Educación popular. Un acercamiento a una práctica libertaria. https://www.nodo50.org/pretextos/educ1.htm 


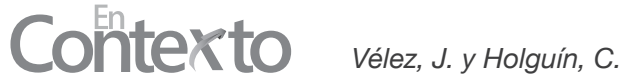

Restrepo, J., Insuasty,A., y Palacios, J. (2015). Círculos solidarios una metodología de economía alternativa en Medellín. En Memorias XX Congreso Internacional de Contaduría, Administración e informática. https://www.researchgate. net/publication/287841438_Circulos_Solidarios_Una_Metodologia_De Economia_Alternativa_En_Medellin

Rodríguez, M., Gallón, N. y Gómez, Y. (2018). Contabilidad Popular. Una propuesta para resignificar las prácticas contables en las organizaciones. Sello Editorial Publicar-T. https://www.tdea.edu.co/index.php/catalogode-publicaciones/107-tdea/sello-editorial/1407-observatorio-publicoexperiencias-tendencias-y-desafios

Rodríguez, M., Gallón-Vargas, N., y Gómez Zapata, Y. (2016). La contabilidad en propuestas de economía solidaria y alternativa. Revista En-Contexto, 4(5), 185-206. https://ojs.tdea.edu.co/index.php/encontexto/article/view/368/386

Torres, A. (1993). La educación popular: evolución reciente y desafíos. Pedagogía y saberes, 4, 14-27. https://revistas.pedagogica.edu.co/index.php/PYS/ article/view/6183

Universidad de Antioquia. (22 de mayo de 2020). ¿Qué es Buppe? Banco Universitario para Programas y Proyectos de Extensión. http://www.udea. edu.co/wps/portal/udea/web/inicio/extension/extension-udea/buppe

\section{Para citar este artículo:}

Vélez, J. y Holguín, C. (2021). Contabilidad popular. Alternativa para resignificar las prácticas contables en las organizaciones sin ánimo de lucro. Estudio de caso. En-Contexto, 9(14), 201-222. 